\title{
Automatic Number Plate Recognition (ANPR) With E-challan using Super Resolution Algorithm
}

\author{
Prof Supriya Thombre ${ }^{1 *}$, Rakshanda Tarekar², Sakshi Ramgirwar ${ }^{3}$, \\ Shivani Zulkanthiwar ${ }^{4}$ and Shubhra Dubey ${ }^{5}$ \\ ${ }^{*}$ Assistant Professor, Dept. of Computer Technology, Yeshwantrao \\ Chavan College of Engineering, Nagpur, Maharashtra, India \\ 2,3,4,5 Students, Dept. of Computer Technology, Yeshwantrao Chavan College \\ of Engineering, Nagpur, Maharashtra, India
}

\section{ABSTRACT}

Number Plate Recognition is most efficient and intelligent system to find the location of the number plate by analyzing image/video data for automatic number plate recognition system (ANPR). Images /videos are captured by a camera placed at every intersection of the road. For identification, the super-resolution techniques are to be applied to the image so that the license plate will get easily identified with the help of OCR technique. The nature and texture of an image should be maintained during the super-resolution of an image. After getting vehicle number it will initiate the activity to generate challan and send the email to the vehicle owner.

KEY WORDS: AUTOMATIC NUMBER PLATE RECOGNITION, ANPR, E-CHALLAN, SUPER RESOLUTION, LICENSE PLATE DETECTION, RULE VIOLATION DETECTION.

\section{INTRODUCTION}

Nowadays, every city is converting into a smart city and people's demand for cars is growing and so are the problems, such as parking lot management, traffic rules violation, etc. The percentage of accidents are increasing now-a-days due to increasing vehicles on road and breaking the traffic rules by many vehicles. For reducing the traffic accidents and to avoid traffic violations for ensuring the safety the images from the installed cameras have been captured. In our country, we have fewer traffic cops and laws associated with traffic regulation are not as strict as in other countries. Sometimes, the rule

\section{ARTICLE INFORMATION}

*Corresponding Author: supriyathombre@gmail.com Received 14th Oct 2020 Accepted after revision 29th Dec 2020 Print ISSN: 0974-6455 Online ISSN: 2321-4007 CODEN: BBRCBA

Thomson Reuters ISI Web of Science Clarivate Analytics USA and Crossref Indexed Journal

\section{Clarivate
Analytics}

NAAS Journal Score 2020 (4.31)

A Society of Science and Nature Publication,

Bhopal India 2020. All rights reserved.

Online Contents Available at: http//www.bbrc.in/

Doi: http://dx.doi.org/10.21786/bbrc/13.14/54 violators are left unseen by the traffic police due to lack of concentration or they are not able to catch them due to which no challan is generated against them. We can solve this problem with the help of the Number Plate Recognition System by using the captured image.

Literature Survey: M. Chaitanya Sai, Deepesh Chandwani, and Saravana Bhava proposed a method that uses the Otsu Thresholding scheme. The image was firstly segmented by the proposed methodology and double binarized for object detection and license plate detection. The blurred or noisy image has been used during the implementation and the multi-object detection is used for license plate detection. The implementation has carried out using multiple stages like the acquisition of an image, gray processing, binarization of an image, recognition of number plate, and OCR is used for template matching. For checking the performance of an algorithm, the authors have used various real-time test images for extracting the number plates from an inputted image. The IoT can be used along with the proposed approach for enhancing the tracking systems [M Chaitanya Sai, 2019].

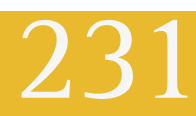


The two approaches have been used by an Automated License Plate Recognition (ALPR) for performing license plate detection (LPD) and perform license plate recognition (LPR). The LPD uses character segmentation and optical character recognition (OCR) for identifying vehicle location and license plate detection. As an alternative of using a general object detector method like Faster-RCNN or SSD300, the authors have used segmentation free OCR evaluation approach for developing a precise system for detecting license plates. The detection in the feature map pyramid is performed by object detectors for detecting objects at different scales. As the deep network needs multiple samples, the new data augmentation technique has been developed which has the potential to train and evaluate [Jaskirat Singh, 2019].

The authors have identified the problems during image capturing. The vehicle license plate detection from an image is itself a complex problem as the image is captured can contain noise. For considering complex scenarios, the application demands robust and generalized license plate detection techniques. The new technique has been proposed by the authors which mainly focuses on the problems such as variations in illumination and color pattern of an image. They have used a static camera for capturing an image. The proposed method can be used for the detection of license plates in each frame of a video sequence, detecting license plates partially and the detection of license plates using moving cameras and vehicles. For object detection, region proposal (RCNN) with convolutional neural networks and its inheritors (Fast-RCNN and Faster-RCNN), and the exemplar-SVM have been used [R Naren Babu, 2019] [Haixiang Li, Ran Yang, 2018].

Yogiraj Kulkarni, Shubhangi Bodhke, et.el considers moving object for license plate detection. The proposed method uses a neural network with an improved Optical Character Recognition (OCR). This method uses K-Nearest Neighbors (KNN) for classifying the features retrieved from region properties in an image. This system uses a decision tree classifier with Ada-Boost for detecting a partial and complete helmet. For achieving accuracy, they have also used CNN for classification of the pretrained model. For subtracting the background from a video for getting moving objects, various features of computer vision have been used like Hough transform, LBP, HOG, SIFT, etc. For identifying geometric shapes like circles, ellipses in an image Hough transform is used. The distribution of edge directions or intensity gradients is used for describing the shape and its appearance of a local object within an image. For describing the local texture structure and for capturing key-points in an image the LBP and SIFT have been used respectively. The author gets an accuracy of 98.72\% for traffic videos which was earlier as $96.36 \%$ using Tesseract OCR on the test images [Yogiraj Kulkarni, 2018].

Praveen and Arihant have significantly shown the usage of deep learning techniques for simplifying the process of ALPR which also allows the inclusion of all the abnormal features present in the license plates. The proposed method uses a recursive training method for the character segmentation network and for detecting the license plate. The same data have been used for training the YOLO framework. This network has proven for fast object detection in a real-time scenario [Praveen Ravirathinam 2020]. A recursively region-growing procedure with some heuristic approach is used by Region Growing Algorithm (RGA). This also focuses on the symbols present on the license plate. The background of the license plate consists of region dimensions, and position, width, and height (aspect ratio), and color and typestyle size. The proposed algorithm is used for identifying the Region of Interest (ROI). This algorithm has been tested on 350 images containing light and heavy vehicles [Sarthak Babbar, 2018].

The license plate recognition (LPR) algorithm is based on license plate region extraction, plate character segmentation, and each character recognition. Image acquisition is a very challenging task due to a variety of number plates formats and the non-uniform lighting conditions. Taking this into consideration many approaches works for limited conditions such as vehicle speed limit, fixed lightning, selected routes, and stationary backgrounds. Immobile images or video sequences have been used in various techniques developed for LPR. The authors have carried out the work categorizing and assessing them by considering the time required for processing, the computational power, and recognition rate [Aishwarya Menon, 2018].

\section{Proposed Methodology}

A) Overview: Automatic Number Plate Recognition (ANPR) is a system which itself a capable for license plate detection without human intervention. It uses an image captured with supporting illumination.

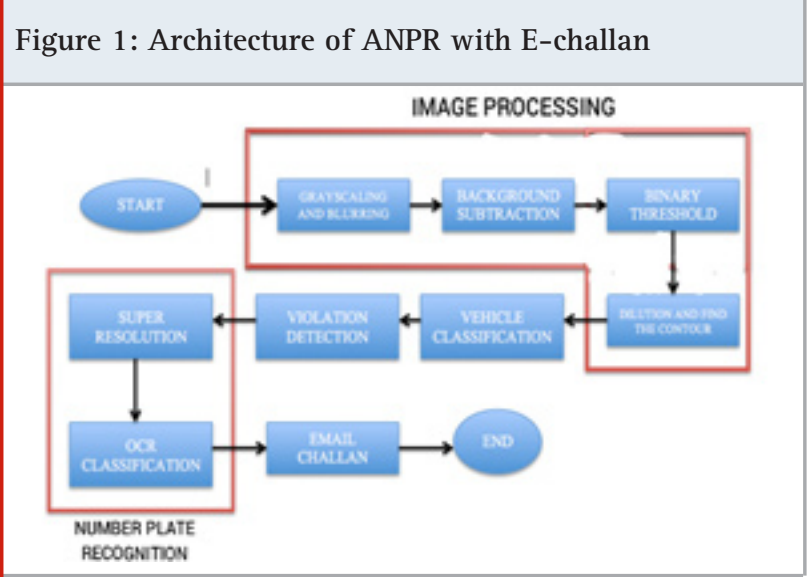

Figure 1 shows the flowchart of the process. When an image is considered for license plate detection it undergoes four factors, such as gray scaling and blurring, background subtraction, binary threshold, dilution, and find the contour. After which the vehicle classification and detection is done where the rule violating vehicles are classified. For Number plate recognition, Superresolution and OCR classification is used. Once the license plate is detected for a particular vehicle, an 
email is generated and sent using the SMTP service to the registered email-id.

\section{B) Image processing}

1. Grayscaling and Blurring: As the part of preprocessing the input frame got from the CCTV footage, the image is grayscaled and blurred with the Gaussian Blur method.

2. Background Subtraction: The background subtraction method is used to subtract the current frame from the reference frame to get the desired object's area. The equation shows the method. $\operatorname{dst}(\mathrm{I})=$ saturate $(\mid \operatorname{scr} 1(\mathrm{I})$ - scr2(I)|)

3. Binary Threshold: The binarization method is used to remove all the holes and noises from the frame and get the desired object area accurately.

4. Dilation and find the contour: After getting the thresholded image, it is dilated to fill the holes, and the contour is found from the image and it is denoted by the rectangle box.

C) Vehicle classification: From the preprocessed, image moving objects are extracted. A vehicle classification model, Mask RCNN is used to classify those moving objects into three class - Car, Motobike, and Non-vehicle. For solving segmentation problems in computer vision, Mask RCNN- a deep neural network can be used. The classifier model is built with MobileNet v1 Neural Network Architecture. In figure 2, the green squares are the bounding boxes.

D) Violation Detection: This is performed with the help of the comparison algorithm. With the help of Mask RCNN, the vehicle classification and a threshold line are made at the signal or parking lots. Whichever car crosses the line will be considered as the rule violator i.e. in figure 2 , the red line indicates the threshold line, and the vehicles in red boxes are the ones who broke the signal.

Figure 2: Vehicle Classification and Violation Detection

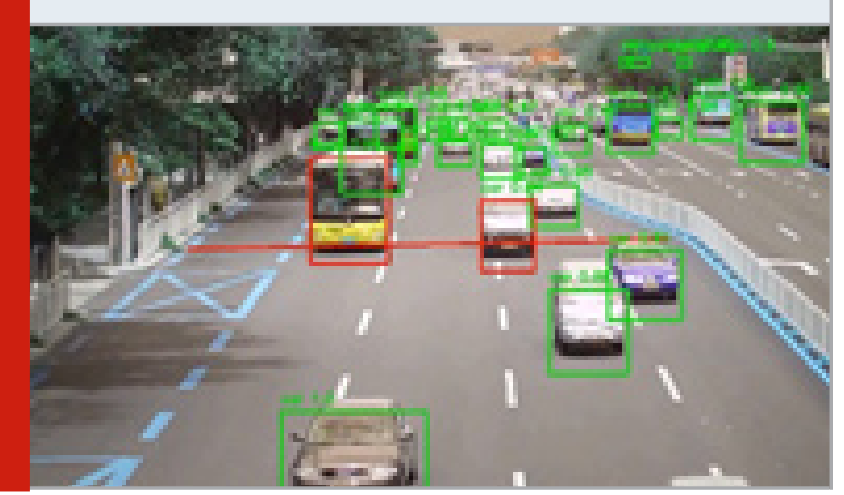

E) Number plate recognition: The number plate recognition consists of the following steps:

1. Super-Resolution: Super resolution uses a combination of a low-resolution sequence of images of a scene for generating a high-resolution image. The high-resolution image can be reconstructed from the low-resolution images.
The Fast-Super-Resolution Convolutional Neural Network (FSRCNN) approach for super-resolution has been used. This approach has mainly 5 steps:

a. Feature Extraction: Adopts the original low-resolution image as input.

b. Shrinking

c. Mapping

d. Expanding

e. De-convolution: Gives the higher resolution image.

The proposed approach will generate a high-resolution image in a lesser time than the existing methods. This method can also be implemented in the real-time images.

2. Optical Character Recognition (OCR): It is a process in which an electronic device is used for checking the characters, shapes by detecting the color pattern present on the paper and translate it into machine-encoded text. This is generally used for converting the text image into a text. While generating a high-resolution image from its corresponding low-resolution image it may produce multiple errors due to a lack of high-frequency image details in low-resolution text images which may lead to incorrect information retrieval. For achieving the higher OCR accuracy, the super-resolution preprocessing on inputted text images should be carried out.

F) Email Challan: The challan will be generated based on violations by vehicles and an e-challan will be sent via email to the violator using SMTP service. To achieve this the Simple Mail Transfer Protocol (SMTP) Server can be used. By using this SMTP, the system can easily send or receive emails. It acts as a relay between the sender and receiver of the email.

\section{Applications}

1. Traffic controlling - This system can be used for controlling the traffic especially in those areas where chances of crimes are more.

2. Parking control - This will help in no parking areas, where sometimes people park their vehicles causing issues like traffic jams.

3. Paperwork reduction - This system is directly sending an e-challan on the registered mobile number as well as on the registered email address. So, the paperwork can be saved.

\section{CONCLUSION}

It has been observed that the images captured from the web cameras installed at every traffic signal provide the blurred and noisy image with poor quality due to the distance and the climate conditions. The proposed method improves the quality of an image using image super-resolution techniques and will also detect the license plate of the vehicle. The rule violator can be easily detected, and the e-challan will be sent on his registered phone no as well as on the email address. 


\section{REFERENCES}

Abhay Singh, Anand Kumar Gupta, Anmol Singh, Anuj Gupta, Sherish Johri, "Vehicle number plate detection using image processing”, published in March 2018 in International Research Journal of Engineering and Technology (IRJET).

Aishwarya Menon, Bini Omman, "Detection and recognition of multiple license plate from still images", published in 1st November 2018 at international conference on systems in digital enterprise technology.

Chi-Hung Chuang, Luo-Wei Tsai, Ming-Shan Deng, Jun-Wei Hsieh, Kuo-Chin Fan, "Vehicle Licence Plate Recognition using Super-Resolution Technique", published in 2014, 11th IEEE International Conference on Advanced video and signal-based surveillance (AVSS).

Haixiang Li, Ran Yang, Xiaohui Chen, "Licence plate detection using convolutional neural network and visual features”, published on 19th November 2018 in 2017 at 3rd IEEE international conference on computer and communications.

Jaskirat Singh, Bharat Bhushan, "Real-time automatic license plate recognition through deep multitask network", published on 17th January 2019 at computing communication and intelligent systems (ICCCIS) International conference.

Jin Chang, Chen Tianhua, Ji Linhao, "Vehicle license plate recognition based on edge detection algorithm", published in 8May 2020 in International conference on intelligent information hiding and multimedia signal processing.

M Chaitanya Sai, Beepesh Chandwani, “Advanced vehicle monitoring system with multi-object automatic number plate detection", published on 18-20 0ctober 2019 at global conference for advancement in technology (GCAT).

Nay Mtet Lin, Yan Lin Aung, Win Kay Khaing, "Automatic vehicle license plate recognition system for smart transportation”, published on 7th January 2019 at IEEE international conference on internet of things and intelligent system (IoTAIS).

Praveen Ravirathinam and Arihant Patamari, "Automatic license plate recognition for indian roads using faster-RCNN", published on 7th May 2020, 20th December 2019 at international conference on advanced computing (ICOAC) at Chennai.

R Naren Babu, V Sowmya and K P Soman, "Indian car number plate recognition using deep learning", published on 13th February 2019 at international conference on intelligent computing, instrumentation and control technology.

Sarthak Babbar, Saowmya Kesarwani, Navroz Dewan, Kartik Shangle, Sanjeev Patel, "A new approach for vehicle number plate detection", published in 12th november 2018 at eleventh internation conference on contemporary computing.

Yogiraj Kulkarni, Shubhangi Bodhke, Amit Kamthe, Archana Patil, "Automatic number plate recognition for motorcyclists riding without helmet”, published on 29th November 2018 at international conference on current trends towards converging technologies (ICCTCT). 